\title{
Gauge-Boson Masses and Mixings in Left-Right-Symmetric Models
}

\author{
E. Massó(a) \\ Stanford Linear Accelerator Center, Stanford University, Stanford, California 94305 \\ (Received 29 March 1984)
}

\begin{abstract}
This paper reports a study of $\mathrm{SU}(2)_{L} \otimes \mathrm{SU}(2)_{R} \otimes \mathrm{U}(1)_{B-L}$ gauge models with a completely general Higgs sector. The scalars which are consistent with the known low-energy phenomenology are determined. The results are used to obtain useful constraints on the $W_{L}-W_{R}$ mixing angle, $\xi$. In particular, the stringent upper limit $\xi \leqslant 0.002$ is derived.
\end{abstract}

PACS numbers: $14.20 . \mathrm{Er}, 11.30 . \mathrm{Rd}, 12.10 . \mathrm{Ck}$

The standard $\mathrm{SU}(2)_{L} \otimes \mathrm{U}(1)$ gauge model $^{1}$ can accommodate all the present electroweak data. The recent discovery ${ }^{2}$ of the weak gauge bosons at CERN, with average masses ${ }^{3} M_{W}=80.9 \pm 2.0 \mathrm{GeV}$ and $M_{Z}=93.0 \pm 2.0 \mathrm{GeV}$, confirmed the validity of the standard model at low energies. These masses, in conjunction with the extrapolated and corrected value of the weak mixing angle, ${ }^{4-6} \sin ^{2} \theta^{W, Z}$ $=0.217 \pm 0.014$, and with the definition

$$
\rho \equiv M_{W}^{2} / M_{Z}^{2} \cos ^{2} \theta^{W, Z}
$$

yield $\rho=0.97 \pm 0.07$. Similarly, the preliminary results reported by Geweniger ${ }^{7}$ lead to $\rho=0.99$ \pm 0.07 . This is consistent with the measurements of the neutral-current strength, ${ }^{4}$ and is to be compared to the prediction $\rho=1$, obtained in the standard theory when the Higgs fields are doublets of the $\mathrm{SU}(2)_{L}$ group. In contrast, Higgs fields with higher weak isospin give $\rho \neq 1 .^{8}$ If we exclude unnatural cancellations, the dominance of Higgs doublets in the $\mathrm{SU}(2)_{L} \otimes \mathrm{U}(1) \rightarrow \mathrm{U}(1)_{\text {e.m. }}$. breaking is strongly favored by experiment.

The standard model, however, has many open theoretical questions. Some of them can be understood by going to enlarged theories. An appealing extension of the standard model is the $\mathrm{SU}(2)_{L}$ $\otimes \mathrm{SU}(2)_{R} \otimes \mathrm{U}(1)_{B-L}$ gauge theory, ${ }^{9-13}$ which represents the minimal incorporation of the leftright symmetry at high energies. In this model, the spontaneous breaking of parity symmetry provides a deeper understanding ${ }^{9}$ of the $V-A$ weak structure observed at low energies. In addition, the scale of parity restoration can be related to the neutrino mass, ${ }^{10}$ as well as to the magnitude of some $C P$ nonconservation effects. ${ }^{11}$ Furthermore, the $\mathrm{SU}(2)_{L} \otimes \mathrm{SU}(2)_{R} \otimes \mathrm{U}(1)$ model could be an effective stage in the breaking of some grandunification groups. ${ }^{12}$ These attractive features motivate a great deal of interest in these theories ${ }^{13}$ and make worthwhile serious experimental and theoretical efforts to constrain left-right-symmetric models as much as possible.

In the spectrum of these models one has elemen- tary Higgs fields, which spontaneously generate fermion and gauge-boson masses. Usually, one chooses the minimal Higgs sector which makes the low-energy limit of the model consistent with experiment. ${ }^{10,13}$ Although this choice can be justified from the point of view of simplicity, it certainly represents one of the arbitrary features of the theory. Indeed, an enlarged scalar spectrum might also be consistent with the available experimental data. Obviously, these data constrain the symmetry properties of the Higgs fields and the pattern of symmetry breaking. The main purpose of the present note is to study systematically these constraints.

Let us introduce a generic set of Higgs fields, $\phi_{\alpha} \sim\left(I_{L}, I_{R}, Y\right)$, where $I_{L}\left(I_{R}\right)$ is the left (right) weak isospin and $Y=B-L$ is the hypercharge. The index $\alpha$ denotes the $I_{L}, I_{R}, Y$ quantum numbers and, eventually, distinguishes Higgs fields with the same symmetry properties. Gauge boson masses arise from the Lagrangian term

$$
\begin{aligned}
& \mathscr{L}=\sum_{\alpha} \operatorname{Tr}\left(D^{\mu} \phi_{\alpha}\right)^{\dagger}\left(D_{\mu} \phi_{\alpha}\right) \\
& \begin{aligned}
D^{\mu} \phi_{\alpha}=\partial^{\mu} \phi_{\alpha} & -i g \overrightarrow{\mathrm{W}}_{L}^{\mu} \cdot \overrightarrow{\mathrm{L}} \phi_{\alpha} \\
& -i g \phi_{\alpha} \overrightarrow{\mathrm{R}} \cdot \overrightarrow{\mathrm{W}}_{R}^{\mu}-i\left(g^{\prime} / 2\right) Y \phi_{\alpha} B^{\mu} .
\end{aligned}
\end{aligned}
$$

In the covariant derivative, $\overrightarrow{\mathrm{L}}$ and $\overrightarrow{\mathrm{R}}$ are the matrix representations according to which the Higgs transform under $\mathrm{SU}(2)_{L}$ and $\mathrm{SU}(2)_{R}$ gauge rotations, respectively. If we denote by $\mu^{2}\left(A-A^{\prime}\right)$ the gauge boson mass term associated with $A_{\mu}^{\dagger} A^{\prime \mu}$, the elements of the $5 \times 5$ mass matrix read

$$
\begin{aligned}
& \mu^{2}\left(W_{L}^{i}-W_{L}^{i}\right)=g^{2} l_{i}, \\
& \mu^{2}\left(W_{R}^{i}-W_{R}^{i}\right)=g^{2} r_{i}, \\
& \mu^{2}\left(W_{L}^{1}-W_{R}^{j}\right)=g^{2} m_{i j}, \\
& \mu^{2}(B-B)=g^{\prime 2}\left(l_{3}+r_{3}+2 m_{33}\right), \\
& \mu^{2}\left(W_{L}^{3}-B\right)=-g g^{\prime}\left(l_{3}+m_{33}\right), \\
& \mu^{2}\left(W_{R}^{3}-B\right)=-g g^{\prime}\left(r_{3}+m_{33}\right),
\end{aligned}
$$




$$
\begin{aligned}
& \text { with } i=1,2,3, \text { and } \\
& m_{i j}=\sum_{\alpha} \operatorname{Tr}\left\langle R_{j} \phi_{\alpha}^{\dagger} L_{i} \phi_{\alpha}\right\rangle, \\
& l_{i}=\Sigma_{\alpha} \operatorname{Tr}\left\langle\left(L_{i}\right)^{2} \phi_{\alpha} \phi_{\alpha}^{\dagger}\right\rangle, \\
& r_{i}=\sum_{\alpha} \operatorname{Tr}\left\langle\left(R_{i}\right)^{2} \phi_{\alpha}^{\dagger} \phi_{\alpha}\right\rangle .
\end{aligned}
$$

In these results, the brackets indicate that the vacuum expectation value of the Higgs fields has been taken. We have used $\langle Q \phi\rangle=0$, where the charge operator $Q$ is the usual combination of gauge generators, namely, $Q=T_{3 L}+T_{3 R}+T_{Y} / 2$.

The structure of the mass matrix reveals that, to suppress right-handed currents and left-right mixings at low energies, parity must be broken by lefthanded singlets, $\Delta_{\beta}^{R} \sim(0, I, Y \neq 0)$, acquiring vacuum expectation values much higher than the $W_{L^{-}}$ mass scale, i.e.,

$$
\frac{\sum_{\alpha} \operatorname{Tr}\left\langle\chi_{\alpha} \chi_{\alpha}^{\dagger}\right\rangle}{\sum_{\beta}\left\langle\Delta_{\beta}^{R}\left(\Delta_{\beta}^{R}\right)^{\dagger}\right\rangle}=O(\eta)<<1 .
$$

Here, $\eta=M_{W_{L}}^{2} / M_{W_{R}}^{2}$ and the $\chi_{\alpha}$ are scalars with $I_{L} \neq 0$, inducing the standard $\mathrm{SU}(2)_{L} \otimes \mathrm{U}(1)$ $\rightarrow \mathrm{U}(1)_{\text {e.m. }}$ transition. In the limit $\eta<<1$, the neutral eigenstates of the mass matrix are

$$
\begin{aligned}
& Z_{L}^{\mu}=\cos \theta W_{L}^{3 \mu}-\sin \theta \tan \theta W_{R}^{3 \mu}-\sigma \tan \theta B^{\mu}, \\
& Z_{R}^{\mu}=(\sigma / \cos \theta) W_{R}^{3 \mu}-\tan \theta B^{\mu}, \\
& A^{\mu}=\sin \theta\left(W_{L}^{3}+W_{R}^{3}\right)^{\mu}+\sigma B^{\mu},
\end{aligned}
$$

where $\sin ^{2} \theta=g^{\prime 2} /\left(g^{2}+2 g^{\prime 2}\right), \sigma^{2}=\cos 2 \theta$, and $A^{\mu}$ is the photon field. Now, the current associated with the neutral $Z_{L}$ boson is

$$
J_{\mu}=(g / \cos \theta)\left(J_{L \mu}^{3}-\sin ^{2} \theta J_{\mu}^{\text {e.m. }}\right) .
$$

On identification of $\theta$ with the standard weak angle, Eq. (4) is just the successful standard neutral current. In other words, $\mathrm{SU}(2)_{L} \otimes \mathrm{SU}(2)_{R}$ $\otimes U(1)$ models with a general scalar spectrum, implemented with the hierarchy given by Eq. (3) and with $\theta=\theta_{W}$, have a low-energy neutral-current phenomenology in agreement with experiment. The reason is that, for $\eta<<1$, the eigenstates of the mass matrix are independent of the details of the Higgs sector. However, the mass eigenvalues depend on the $l, r$, and $m$ parameters. Indeed, the right-handed boson masses carry relevant information on the isospin of the $\Delta^{R}$ scalars. More interesting for our purposes is the left-handed boson sector. Calculating the masses and introducing them in Eq. (1) yields

$$
\rho=\frac{\operatorname{Tr} \sum_{\alpha}(\tilde{L})^{2}\left\langle\chi_{\alpha} \chi_{\alpha}^{\dagger}\right\rangle}{2 \operatorname{Tr} \sum_{\alpha}\left(L_{3}\right)^{2}\left\langle\chi_{\alpha} \chi_{\alpha}^{\dagger}\right\rangle},
$$

where $(\tilde{L})^{2} \equiv(\overrightarrow{\mathrm{L}})^{2}-\left(L^{3}\right)^{2}$. Barring unnatural cancellations, $\rho=1$ is obtained only with $I_{L}=\frac{1}{2}$ Higgs fields. The experimental value of $\rho$ indicates that the spontaneous $\mathrm{SU}(2)_{L} \otimes \mathrm{U}(1)$ breaking in leftright-symmetric theories is strongly dominated by $\chi_{\alpha} \sim\left(\frac{1}{2}, I_{R}, Y\right)$ fields. This result is easy to understand, since that breaking is essentially controlled by the $I_{L}$ isospin of $\chi_{\alpha}$. I remark that present data still have room for the small effects of order $\eta$ which have been neglected in Eqs. (4) and (5).

Let us address now some considerations which will shed more light on the restrictions on the scalar sector. In left-right-symmetric models, the scalar potential is invariant under the transformation $\phi_{\alpha} \leftrightarrow \bar{\phi}_{\alpha}$, where $\bar{\phi}_{\alpha} \sim\left(I_{R}, I_{L}, Y\right)$ is the parityconjugate field of $\phi_{\alpha} \sim\left(I_{L}, I_{R}, Y\right)$. Consider the $\Delta_{\beta}^{L} \equiv\left(\bar{\Delta}_{\beta}^{R}\right) \sim(I, 0, Y)$, with $I \neq \frac{1}{2}$, and the $\chi_{\alpha}$ $\sim\left(\frac{1}{2}, I_{R}, Y\right)$ fields. From Eq. (3) and the fact $\Delta^{L}$ scalars induce $\rho \neq 1$, there follows the hierarchy

$$
\left\langle\left(\Delta_{\beta}^{L}\right)^{\dagger} \Delta_{\beta}^{L}\right\rangle<<v_{R}^{2},
$$

where $v_{R}^{2}=\Sigma_{\gamma}\left(\Delta_{\gamma}^{R}\left(\Delta_{\gamma}^{R}\right)^{\dagger}\right\rangle$. This parity-breaking configuration minimizes the potential. ${ }^{10,13}$ Let us evaluate the order of magnitude of the $\bar{\chi}_{\alpha}$ $\sim\left(I_{R}, \frac{1}{2}, Y\right)$ vacuum expectation values in this configuration. For reasons that will shortly become clear, we first discuss the $I_{R} \neq 0$ case. We exclude the uninteresting fields that do not contribute appreciably to the sum in Eq. (5) and approximate $\operatorname{Tr}\left\langle\chi_{\alpha} \chi_{\alpha}^{\dagger}\right\rangle \simeq k^{2}$ and $\operatorname{Tr}\left\langle\bar{\chi}_{\alpha} \bar{\chi}_{\alpha}^{\dagger}\right\rangle \simeq \bar{k}^{2}$. For our purpose, the relevant part of the potential is given approximately by

$$
\begin{gathered}
V=\left(-\mu^{2}+\lambda_{1} v_{R}^{2}\right)\left(k^{2}+\bar{k}^{2}\right)+\lambda_{2} v_{R}^{2} k \bar{k} \\
+\lambda_{3}\left(k^{4}+\bar{k}^{4}\right)+\lambda_{4} k^{2} \bar{k}^{2},
\end{gathered}
$$

where the $\lambda_{i}$ are linear combinations of Yukawa couplings. The conditions for the minimum imply either $\vec{k} \simeq k$ or

$$
\bar{k} \simeq \frac{\lambda_{2}}{2\left(2 \lambda_{3}-\lambda_{4}\right)} \frac{v^{2} R}{k} .
$$

Provided the $\lambda_{i}$ are not unnaturally large or small, Eq. (6) yields $\vec{k} \gg v_{R}$. Now, the $\rho=1$ requirement for the $\bar{\chi}_{\alpha}$ field is inconsistent with both solutions, unless $\chi_{\alpha} \sim\left(\frac{1}{2}, \frac{1}{2}, Y\right)$. Indeed, $I_{R} \neq \frac{1}{2}$ requires $\bar{k}<<k$, which is far from being satisfied at the minimum of the potential. We notice that the argument is not valid if $\chi_{\alpha}$ has $I_{R}=0$, for then $\bar{\chi}_{\alpha}$ is a $\Delta^{R}$-type scalar.

We conclude that the present low-energy data strongly restrict the Higgs sector in $\mathrm{SU}(2)_{L}$ $\otimes \mathrm{SU}(2)_{R} \otimes \mathrm{U}(1)_{B-L}$ models. The Higgs spectrum which acquires relevant vacuum expectation 
values and leads to the correct low-energy phenomenology is composed of (i) $\mathrm{SU}(2)_{R}$ $\otimes \mathrm{U}(1)_{B-L}$ - breaking $\Delta_{\beta}^{R} \sim(0, I, Y)$ fields, and (ii) $\mathrm{SU}(2)_{L} \otimes \mathrm{U}(1)$-breaking $\quad \chi_{\alpha} \sim\left(\frac{1}{2}, \frac{1}{2}, Y=0, \pm 2\right)$ and $\chi_{\alpha} \sim\left(\frac{1}{2}, 0, Y= \pm 1\right)$ fields; the former, when $Y=0$, also generate charged fermion masses. The $\Delta_{\beta}^{L} \sim(I, 0, Y), I \neq \frac{1}{2}$, scalars do not generate substantial gauge-boson masses. For completeness, we recall that the minimal Higgs sector used in the literature $^{13}$ corresponds to the three scalars $\Delta^{R}=\bar{\Delta}^{L} \sim(0,1,2)$ and $\chi \sim\left(\frac{1}{2}, \frac{1}{2}, 0\right)$.

We can now deduce a useful constraint on the $W_{L}^{ \pm}-W_{R}^{ \pm}$mixing angle, $\xi$, which is induced by the (charged) nondiagonal term in Eq. (2). Among our restricted Higgs fields, only the $\chi_{\alpha} \sim\left(\frac{1}{2}, \frac{1}{2}, Y\right)$ type may connect $W_{L}^{ \pm}$with $W_{R}^{ \pm}$. These scalars fulfill

$$
\sum_{\alpha} \operatorname{Tr}\left\langle\left(\tau_{1}\right)^{2} \chi_{\alpha} \chi_{\alpha}^{\dagger}\right\rangle \geqslant\left|\sum_{\alpha} \operatorname{Tr}\left\langle\tau_{-} \chi_{\alpha}^{\dagger} \tau_{+} \chi_{\alpha}\right\rangle\right|,
$$

where $\tau_{i}$ are the Pauli matrices and $\tau_{ \pm}=\left(\tau_{1}\right.$ $\left.\pm i \tau_{2}\right) / \sqrt{2}$. This tells us that the mass matrix elements induced by $\chi_{\alpha}$ obey $\mu^{2}\left(W_{L}^{ \pm}-W_{L^{ \pm}}\right)$ $\geqslant\left|\mu^{2}\left(W_{L}^{ \pm}-W_{R}^{ \pm}\right)\right|$. The other Higgs representations will certainly contribute to the $W_{L}$-mass generation. It follows that the mixing angle is bounded by

$$
|\xi| \simeq\left|\mu^{2}\left(W_{L}^{ \pm}-W_{R}^{ \pm}\right)\right| / M_{W_{R}}^{2} \leqslant \eta .
$$

This bound was noticed ${ }^{14}$ in the context of the minimal Higgs sector. Now I claim that Eq. (7) constitutes a completely general constraint (arising in fact from low-energy requirements) involving two of the fundamental parameters of left-rightsymmetric theories.

We finally consider some phenomenological implications of Eq. (7). To reach our bound we have made no assumptions about the $U_{R}$ mixing matrix appearing in the charged right-handed current. However, the usual analyses ${ }^{15}$ essentially assume either $U_{R}=U_{L}$ or $U_{L}^{*}$, with $U_{L}$ being the lefthanded mixing matrix. Let us quote the most stringent published bounds on $\xi$ and $\eta$. On the one hand, nonleptonic $\Delta S=1$ decays yield ${ }^{16}|\xi| \leqslant 4$ $\times 10^{-3}$. This limit, unfortunately, is subject to the theoretical uncertainties associated with nonleptonic processes. The safer semileptonic decays and the bottom lifetime measurements have been recently used $^{17}$ to reach $|\xi| \leqslant 5.5 \times 10^{-3}$, obtained if one includes a $5 \%$ fractional theoretical error in $\left(U_{L}\right)_{u s}$. due to $\mathrm{SU}(3)$ symmetry breaking and radiative corrections when analyzing semileptonic hyperon and $K_{e 3}$ decays. ${ }^{18}$ A $10 \%$ error leads to $|\xi| \leqslant 8 \times 10^{-3}$. On the other hand, the analyses ${ }^{14,19}$ of the $K^{0}-\bar{K}^{0}$ transition yield $\eta \leqslant \frac{1}{430}$. This bound has been confirmed by a variety of hadronic models ${ }^{20}$ and eventual cancellations with neutral Higgs mediated transitions are ruled out. ${ }^{21}$ Since QCD corrections $^{22}$ reduce the quoted limit by at least a factor of $3, \eta \leqslant \frac{1}{430}$ has to be considered as a conservative bound. Using this value in Eq. (7) we obtain

$$
|\xi| \leqslant 2.3 \times 10^{-3}
$$

which represents the most stringent constraint now available. The introduction of QCD effects would yield $|\xi| \leqslant 8 \times 10^{-4}$.

If left-right symmetry is present at high energies, a careful analysis of weak low-energy transitions could in the near future detect the presence of the mixing between left- and right-handed currents. An eventual determination of a nonvanishing $\xi$ would reveal, through Eq. (7), the range of energies where parity should be restored. There is already a claim ${ }^{23}$ of a significant departure of $\xi$ from zero. Unfortunately, the analysis is rather model dependent and we cannot infer with confidence a lower bound on $\eta$.

Discussions with J. Bagger, F. Gilman, H. Reno, and G. Senjanović are gratefully acknowledged. This work was supported in part by the U. S. Department of Energy under Contract No. DEAC03-76ER00515, and by the Fulbright/Spanish Ministerio de Educación y Ciencia Commission.

(a) On leave of absence from Departament de Física Teòrica, Universitat Autònoma de Barcelona, Bellaterra, Spain.

${ }^{1}$ S. L. Glashow, Nucl. Phys. 22, 579 (1961); A. Salam, in Elementary Particle Theory, edited by N. Svartholm (Almqvist and Wiksells, Stockholm, 1969); S. Weinberg, Phys. Rev. Lett. 19, 1264 (1967).

${ }^{2}$ G. Arnison et al. (UA1 Collaboration), Phys. Lett. 129B, 273 (1983), and 126B, 398 (1983); P. Bagnaia et al. (UA2 Collaboration), Phys. Lett. 129B, 130 (1983); M. Banner et al., Phys. Lett. 122B, 476 (1983).

${ }^{3} \mathrm{C}$. Rubbia, in Proceedings of the International European Physical Society Conference on High Energy Physics, Brighton, 1983, edited by J. Guy and C. Costain (Rutherford Appleton Laboratory, Chilton, Didcot, Oxfordshire, England, 1983).

${ }^{4}$ J. E. Kim, P. Langacker, M. Levine, and H. H. Williams, Rev. Mod. Phys. 53, 211 (1981).

${ }^{5}$ W. Marciano and A. Sirlin, Nucl. Phys. B189, 442 (1981); C. Llewellyn Smith and J. Wheater, Phys. Lett. 105B, 486 (1981).

${ }^{6}$ W. Marciano, Phys. Rev. D 20, 274 (1979); F. Antonelli, M. Consoli, and G. Corbo, Phys. Lett. 91B, 90 (1980); M. Veltman, Phys. Lett. 91B, 95 (1980).

${ }^{7} \mathrm{C}$. Geweniger, in Proceedings of the International European Physical Society Conference on High Energy Physics, 
Brighton, 1983, edited by J. Guy and C. Costain (Rutherford Appleton Laboratory, Chilton, Didcot, Oxfordshire, England, 1983).

${ }^{8}$ D. A. Ross and M. Veltman, Nucl. Phys. B 95, 135 (1975).

9J. C. Pati and A. Salam, Phys. Rev. D 10, 275 (1974); G. Senjanović and R. N. Mohapatra, Phys. Rev. D 12, 1502 (1975).

${ }^{10}$ G. Senjanović and R. N. Mohapatra, Phys. Rev. D 23, 165 (1981).

${ }^{11}$ R. N. Mohapatra and J. C. Pati, Phys. Rev. D 11, 566 (1975); D. Chang, Nucl. Phys. B214, 435 (1983).

${ }^{12} \mathrm{H}$. Georgi, in Particles and Fields - 1974, edited by C. E. Carlson, AIP Conference Proceedings No. 23 (American Institute of Physics, New York, 1975); H. Fritzsch and P. Minkowski, Ann. Phys. (N.Y.) 93, 193 (1975).

${ }^{13}$ For reviews of left-right symmetry, see G. Senjanović, Nucl. Phys. B153, 334 (1979); R. N. Mohapatra, in Proceedings of the NATO Summer School on Particle Physics, Munich, 1983 (to be published).
${ }^{14} \mathrm{G}$. Beall, thesis, University of California at Irvine, 1982 (unpublished).

${ }^{15}$ See Mohapatra, Ref. 13, for an updated review.

16J. Donoghue and B. Holstein, Phys. Lett. 113B, 382 (1982).

${ }^{17} \mathrm{~L}$. Wolfenstein, Institute of Theoretical Physics, University of California at Santa Barbara Report No. NSF-ITP-83-178, 1983 (to be published).

${ }^{18}$ R. E. Shrock and L.-L. Wang, Phys. Rev. Lett. 41, 1692 (1978).

${ }^{19} \mathrm{G}$. Beall, M. Bender, and A. Soni, Phys. Rev. Lett. 48, 848 (1982); H. Harari and M. Leurer, Fermilab Report No. Pub-83/59-THY, 1983 (unpublished).

20J. Trampetić, Phys. Ref. D 27, 1565 (1983).

${ }^{21}$ F. Gilman and M. H. Reno, Phys. Lett. 127B, 426 (1983), and Stanford Linear Accelerator Center Report No. SLAC-PUB-3238, 1983 (unpublished).

22I. I. Bigi and J. M. Frère, Phys. Lett. 129B, 469 (1983).

${ }^{23}$ B. Holstein and S. Treiman, Phys. Rev. D 16, 2369 (1977) 